\title{
BMJ Open Physical activity among medical students in Southern Thailand: a mixed methods study
}

\author{
Apichai Wattanapisit, Krittanu Fungthongcharoen, Udomsak Saengow, \\ Surasak Vijitpongjinda
}

To cite: Wattanapisit $A$, Fungthongcharoen $\mathrm{K}$, Saengow U, et al. Physical activity among medical students in Southern Thailand: a mixed methods study. BMJ Open 2016;6: e013479. doi:10.1136/ bmjopen-2016-013479

- Prepublication history for this paper is available online. To view these files please visit the journal online (http://dx.doi.org/10.1136/ bmjopen-2016-013479).

Received 15 July 2016 Revised 29 August 2016 Accepted 8 September 2016

CrossMark

School of Medicine, Walailak University, Thasala, Nakhon Si Thammarat, Thailand

Correspondence to Dr Apichai Wattanapisit; apichai.wa@wu.ac.th

\section{ABSTRACT}

Objectives: The study aimed to investigate the prevalence of physical activity $(\mathrm{PA})$ and factors influencing PA behaviours among medical students in Southern Thailand.

Design: The study implemented a mixed methods approach. The sequential design consisted of 2 phases: a survey followed by in-depth interviews.

Setting: The study was conducted in the 3 campuses of a medical school in Southern Thailand. The preclinical students (years 1-3) studied general and basic science subjects at Nakhon Si Thammarat campus. The clinical students (years 4-6) received clinical training and hospital attachments at Trang or Phuket campuses. Total number of students was 285 , with $46-48$ students in each class. The study was conducted from September 2015 to February 2016.

Participants: Participants were medical students, 18 years old and above, from 3 campuses without disabilities or medical conditions which limited their ability to perform PA.

Outcome measures: The prevalence of the recommended levels of $P A$ was measured using Global Physical Activity Questionnaire (GPAQ). The association between the demographic data and the recommended PA levels were analysed by univariate and multivariate analysis. In-depth interviews and thematic analysis were completed to explore PA behaviours.

Results: A total of 279 (response rate $97.9 \%$ ) medical students participated in the study. Approximately half $(49.5 \%)$ of the participants were physically active. The median total energy use was 540 metabolic equivalent-min/week (range 0-5640). Male and preclinical students were more likely to be physically active $(p<0.05)$. Twenty-four in-depth interviews were conducted. Supportive factors included social support from friends and families. Study-related activities and overtime shift work were barriers.

Conclusions: More than half of the medical students have insufficient PA because of study-related activities and overtime shift work. Future studies should focus on finding ways to improve PA in clinical and female students in Thailand.

\section{Strengths and limitations of this study}

- This mixed methods study determined the prevalence of physical activity (PA) and factors influencing PA as well as providing explanations of PA patterns among the medical students in Thailand.

- This study achieved a high response rate.

- This study was based on a single medical school from Thailand and the findings may not be generalisable to other medical schools.

\section{INTRODUCTION}

Physical inactivity is one of the global health problems, which increases overall mortality and morbidity. Worldwide, in 2010, the WHO reported $23 \%$ of adults aged 18 years old and above and $81 \%$ of adolescents aged 11-17 years were insufficiently physically active. ${ }^{1}$ Insufficient physical activity (PA) contributes to 3.2 million deaths each year, ${ }^{1}$ and scientific evidence supports that PA can reduce mortality. ${ }^{2}$ Consequently, the WHO sets a global target of ' $10 \%$ relative reduction in the prevalence of insufficient PA' by 2025 to promote world population's health. ${ }^{1}$ The term, PA, is sometimes used interchangeably with exercise. Nevertheless, PA is defined as any bodily movement produced by skeletal muscles that results in energy expenditure, and exercise is a subset of PA. ${ }^{3}$ The WHO recommends that adults should do at least $150 \mathrm{~min}$ of moderate-intensity aerobic PA weekly. ${ }^{4}$ However, about one-fifth $(17.4 \%)$ of adults around the world are physically inactive. ${ }^{5}$ Meeting the recommended levels of PA depends on several factors included age, sex, weight status, education, and health perception. ${ }^{6-11}$

This study focused on PA behaviours of medical students because it is assumed that medical students are aware of the importance of healthy lifestyles. Nonetheless, knowledge is only a part of expressing behaviours 
and providing health promotion. One of the predicting factors of medical students' health condition is their attitude towards health promotion, illness prevention and exercise. ${ }^{12}$ Moreover, the evidence in medical students suggests that there is a strong association between personal PA behaviours and PA counselling attitudes and practices. ${ }^{13}{ }^{14}$ Some previous studies show medical students have more participation in PA than general populations. ${ }^{15}{ }^{16}$ In Thailand a study shows that physical factors (sex and body weight) and environmental factors (society and culture) are the associated factors of PA behaviours. ${ }^{16}$ However, the study does not explain the reasons of the findings, which requires an exploratory approach using a qualitative methodology.

Therefore, the aims of this study were to determine the prevalence of the WHO recommended levels of PA among the medical students in Southern Thailand and explore the factors influencing their PA behaviours.

\section{METHODS}

\section{Study design}

The study implemented a mixed methods approach. The design consisted of two phases: a survey followed by in-depth interviews. The sequential method was devised to first determine the level of PA and factors associated with PA, followed by the exploration and explanation of the observational findings from the survey.

\section{Context/setting}

The study was conducted in three campuses of School of Medicine, Walailak University, in Southern Thailand (Nakhon Si Thammarat, Trang and Phuket). In all campuses, there were accessible exercises or sport facilities such as fitness centres, basketball fields and tennis courts. The preclinical students (years 1-3) studied the general and basic science at the Nakhon Si Thammarat campus. The clinical students (years 4-6) received clinical training and hospital attachments at the Trang or Phuket campus. The total number of students was 285, with 46-48 students in each class. The study was conducted from September 2015 to February 2016.

\section{Participants}

The medical students in academic year 2015 who were 18 years old and above from the three campuses were invited to participate in the study. Students with disabilities or medical conditions which limited the ability of PA, including movement disorder; muscular weakness; heart disease; uncontrolled pulmonary disease; and uncontrolled medical conditions, were excluded.

\section{Phase 1: survey}

All medical students $(n=285)$ were invited to participate in the study. The questionnaire had two parts. First, information including the medical student's personal profile, including level of education, sex, birth date, body weight and height, student's allowance, place of living and underlying illnesses was gathered. Next PA measurements were gathered using a standard self-report questionnaire, the Global Physical Activity Questionnaire (GPAQ) V.2 (Thai version) to collect the information. The information on PA participation was collected in three domains: activities at work, travelling activities and recreational activities. ${ }^{17}$

The participants were briefed about the study and permission was gained through informed consent with written consent forms. The self-administered questionnaires were provided to the participants, and the questionnaires were gathered by researchers within 3 days. The completeness and accuracy of the information was checked, and clarification was requested by the researchers regarding unclear information.

The baseline characteristics of the participants were reported as frequency, percentage, mean and SD. The energy use of each PA domain was calculated as metabolic equivalent (MET)-min/week. The summation of energy use of work, energy use of travelling and energy use of recreation was 'energy use of overall PA'. The $\geq 600$ MET-min/week of energy use of overall PA was considered as the recommended levels of PA. ${ }^{17}$ The prevalence of the recommended levels of PA was calculated by dividing the number of participants who were engaged in $\geq 600$ MET-min/week of energy use of overall PA with the total number of participants. Univariate analysis of the associations between the demographic factors and the recommended levels of PA was analysed by using the Pearson's $\chi^{2}$ and Fisher's exact test, if appropriate. For multivariate analysis, multiple regression analysis was performed to identify factors that were independently associated with medical students' PA. The associations were considered statistically significant when $\mathrm{p} \leq 0.05$.

\section{Phase 2: qualitative study}

Both the preclinical and clinical students who met the recommended levels of PA ( $\geq 600$ MET-min/week) and did not meet the recommendation $(<600$ MET-min/ week) from phase 1 study were selected by purposive sampling for the in-depth interviews to achieve maximal variations and understand the factors influencing PA behaviours. The number of interviewees depended on the saturation of the data where no new themes emerged from the data.

The early list of interview questions was drafted based on the objectives of the study. The socioecological model, which focused on intrapersonal aspects (knowledge, skills, and attitudes); interpersonal aspects (social networks and social support systems); institutional aspects (social institutions); community aspects (relationships among organisations and institutions); and public policies, was a core component for exploring the supportive factors and barriers to PA behaviours. ${ }^{18}$ Then, questions were created to ascertain characteristics of PA behaviours, opinions on PA, and factors influencing PA behaviours. The interviewer also asked some 
probing questions to find out more detail of data. The in-depth interviews were conducted at the participants' convenience. All interviews were conducted by one researcher (AW) in Thai language because it is the medium of communication in the university in Thailand. The interviews were recorded by using a digital voice recorder. The audio records were transcribed verbatim.

Thematic analysis was completed using the data to identify, analyse and report patterns. The process of the analysis consisted of a review of all transcripts, defining the data (coding) and analysing the data. Initially, sets of Thai transcripts were read independently by two researchers (AW and $\mathrm{KF}$ ). The indexes of terms were generated from each transcript. The coding process started from open coding (developed the new issues) followed by axial coding which involved merging the codes that had been developed. Then, selective coding was performed to merge the various codes to core categories. ${ }^{19}$ Finally, the relevant quotations were translated from Thai to English.

\section{RESULTS}

\section{Phase: 1 survey}

\section{Baseline characteristics}

A total of 279 medical students participated in the study (response rate $97.9 \%) ; 50.5 \% \quad(n=141)$ were preclinical students (response rate 98.6\%); and 49.5\% ( $\mathrm{n}=138)$ were clinical students (response rate 97.2\%). The mean age was $20.93 \pm 1.82$ years, and $63.4 \% \quad(\mathrm{n}=177)$ were women. Most participants, $92.5 \%(\mathrm{n}=258)$, had monthly allowance between 5000 and 14999 Baht, and 90.0\% $(\mathrm{n}=251)$ lived in the campus dormitories. More than half, $59.1 \% \quad(\mathrm{n}=165)$, were of healthy weight. Few students, $9.7 \%(n=27)$, had underlying illnesses (15 allergic rhinitis; 3 controlled asthma; 2 migraine; 1 thalassaemia; and 6 other illnesses) (table 1 ).

\section{PA participation}

The median energy use of overall PA was 540 MET-min/ week (range $0-5640$ ). The preclinical students (median 720 MET-min/week; range 0-5640) had a higher energy use of overall PA compared with the clinical students (median 370 MET-min/week; range 0-4560).

For preclinical and clinical students, the major energy use came from recreational activities. In contrast, the energy use from work and travelling were not common (table 2).

About half, $49.5 \% \quad(\mathrm{n}=138)$, met the recommended levels of PA (physically active). Accordingly, preclinical students were more physically active than clinical students $(58.2 \%, \mathrm{n}=82$ vs $40.6 \%, \mathrm{n}=56 ; \mathrm{p}<0.05)$. The univariate analysis showed that preclinical study and male sex were factors associated with PA $(\mathrm{p}<0.05)$ (table 3$)$. Multivariate analysis found that the clinical students were less active than the preclinical students (OR 0.55; $95 \%$ CI 0.33 to 0.91$)$. Women were also less active than men (OR 0.43; $95 \%$ CI 0.25 to 0.73) (table 3). Body

\begin{tabular}{|c|c|}
\hline Demographic characteristic & n (\%) \\
\hline Age (year) mean (SD) & $20.93(1.82)$ \\
\hline \multicolumn{2}{|l|}{ Level of education } \\
\hline Preclinical & $141(50.5)$ \\
\hline Clinical & $138(49.5)$ \\
\hline \multicolumn{2}{|l|}{ Sex } \\
\hline Male & $102(36.6)$ \\
\hline Female & 177 (63.4) \\
\hline BMI $\left(\mathrm{kg} / \mathrm{m}^{2}\right)$ mean (SD) & $21.79(2.88)$ \\
\hline$<18.5$ & $30(10.8)$ \\
\hline $18.5-22.9$ & $165(59.1)$ \\
\hline 23.0-24.9 & 49 (17.6) \\
\hline$\geq 25$ & $35(12.5)$ \\
\hline \multicolumn{2}{|l|}{ Allowance (Baht/month)* } \\
\hline$<5000$ & $21(7.5)$ \\
\hline 5000-9999 & $195(69.9)$ \\
\hline $10000-14999$ & $55(19.7)$ \\
\hline$\geq 15000$ & $8(2.9)$ \\
\hline \multicolumn{2}{|l|}{ Place of living } \\
\hline Campus & $251(90.0)$ \\
\hline Off campus & $28(10.0)$ \\
\hline \multicolumn{2}{|l|}{ Underlying illness } \\
\hline Yes & $27(9.7)$ \\
\hline
\end{tabular}

mass index, allowance, place of living and underlying illness were not significant factors.

\section{Phase 2: qualitative study}

Twenty-four students (12 active and 12 inactive) participated in the interviews. Among the interviewees, 12 were preclinical students and 12 were clinical students; of which 13 were men and 11 were women. Each interview took between 25 and $50 \mathrm{~min}$. The codes were sorted into three categories of themes, including varying understanding of PA; facilitators to PA; and barriers to PA.

\section{Varying understanding of PA}

The physically active students thought that any activities from work or travelling could not replace exercise while some non-exercisers mentioned that exercise could be substituted by activities at work.

Walking or cycling in daily life cannot replace my regular exercise. It is not comparable, in terms of the intensity.

(Student 8, preclinical year, male)

Even I do not do any planned exercises, I walk a lot in the hospital. If we do not have free time for a 30-minute jogging, we can walk instead. Just increase walking. I feel better, unashamed.

(Student 17, clinical year, female) 
Table 2 Physical activity participation

\begin{tabular}{llllll}
\hline & \multicolumn{2}{l}{ Energy use (MET-min/week) } \\
\cline { 3 - 6 } Level of education & $\mathbf{n}$ & Overall & Work & Travelling & \multicolumn{2}{l}{$\begin{array}{l}\text { Recreation } \\
\text { Median (range) }\end{array}$} & Median (range) & Median (range) \\
\hline Preclinical & 141 & $720(0-5640)$ & $40(0-3840)$ & $0(0-1200)$ & $480(0-3360)$ \\
Clinical & 138 & $370(0-4560)$ & $0(0-2520)$ & $0(0-1680)$ & $240(0-3360)$ \\
Total & 279 & $540(0-5640)$ & $0(0-3840)$ & $0(0-1680)$ & $360(0-3360)$ \\
\hline
\end{tabular}

MET, metabolic equivalent.

Facilitators to PA

\section{Family influences PA behaviours}

Family played different roles in motivating the medical students to be physically active: family culture; family support; family encouragement; and family follow-up.

When I was young, my mother woke me up at 5 o'clock for exercise. I have been familiar with an active lifestyle. Nowadays, my mom always asks me 'are you still exercising?' and warns me to relax after the hard study. My family has a strong influence on my behaviour.

(Student 5, preclinical year, male)

Basically, my family supports me to exercise. My dad likes exercise. My dad and mom always go to walk in the park. When I was a child, I went with them. After my leaving home, we have not gone to exercise together. Anyway, sometimes, I call my dad in the evening, and he tells me that 'mom and I are walking in the park'. My dad also asks me about my activities, 'do you run or play badminton recently?'. It reminds me that I should go to exercise.

(Student 13, clinical year, female)
My parents do not exercise, but they encourage me to exercise.

(Student 11, preclinical year, male)

My overweight is observed by my mother. She will tell me to reduce body weight if I get overweight. My father invites me to bike with him when I stay at home. My parents also give me a budget for the gym payment and always ask me about the regularity of exercise when I call them.

(Student 8, preclinical year, male)

\section{Friend supports PA behaviours}

Friends could encourage medical students to participate in PA and also were exercise partners.

I am so lazy. My friends can motivate me to exercise. Jogging without friends is awful.

(Student 10, preclinical year, female)

It is not only an exercise. It is wonderful to be relaxed and talk with friends.

(Student 13, clinical year, female)

I am still not active at the moment. According to the question 'do I want to exercise?', I wish I could. I will go

Table 3 Univariate analysis and multivariate analysis: associations between medical students' profile and physical activity

\begin{tabular}{|c|c|c|c|}
\hline \multirow[b]{2}{*}{ Factor } & \multicolumn{3}{|c|}{ Recommended level ( $\geq 600$ MET-min/week) } \\
\hline & Crude OR (95\% Cl) & Adjusted OR (95\% Cl) & p Value \\
\hline Level of education (ref: preclinical) & & & $<0.05^{\star}$ \\
\hline Clinical & $0.49(0.30$ to 0.79$)$ & 0.55 (0.33 to 0.91$)$ & \\
\hline Sex (ref: male) & & & $<0.05^{\star}$ \\
\hline Female & $0.32(0.20$ to 0.53$)$ & $0.43(0.25$ to 0.73$)$ & \\
\hline BMI $\left(\mathrm{kg} / \mathrm{m}^{2}\right)$ (ref: <18.5) & & & 0.74 \\
\hline $18.5-22.9$ & 1.42 (0.65 to 3.12$)$ & 1.28 (0.56 to 2.92$)$ & 0.56 \\
\hline 23.0-24.9 & $1.36(0.55$ to 3.34$)$ & $1.08(0.41$ to 2.87$)$ & 0.87 \\
\hline$\geq 25$ & 0.87 (0.32 to 2.34$)$ & 0.85 (0.30 to 2.44$)$ & 0.77 \\
\hline Allowance (Baht/month) $\dagger($ ref: <5000) & & & 0.24 \\
\hline 5000-9999 & $1.52(0.61$ to 3.78$)$ & 1.15 (0.45 to 2.95$)$ & 0.78 \\
\hline $10000-14999$ & $0.96(0.35$ to 2.65$)$ & 0.67 (0.23 to 1.98$)$ & 0.47 \\
\hline$\geq 15000$ & 0.44 (0.07 to 2.74$)$ & 0.38 (0.06 to 2.43$)$ & 0.30 \\
\hline Place of living (ref: campus) & & & 0.26 \\
\hline Off campus & $0.53(0.24$ to 1.20$)$ & $0.62(0.27$ to 1.43$)$ & \\
\hline Underlying illness (ref: no) & & & 0.94 \\
\hline Yes & $0.90(0.41$ to 1.99$)$ & $1.03(0.44$ to 2.41$)$ & \\
\hline
\end{tabular}


if I have some friends to go with.

(Student 19, clinical year, female)

\section{Barriers to PA}

\section{Academic study takes priority}

Some medical students considered study-related activities more important than PAs.

We have an important exam for passing to the clinical years. I have not done my regular exercise as I usual do because I have to study with my group in the evenings.

(Student 5, preclinical year, male)

Being medical students, we have a lot of work, less sleeping time, sedentary lifestyle and unhealthy diet. What a pity we are! I personally think our bodies need exercise.

(Student 12, preclinical year, male)

\section{Heavy clinical workload disrupts exercise routine}

Regular night duties disrupted the students' exercise routine because they felt tired after the night shift.

After a night shift, on the next day, I feel too tired for doing anything-even reading books. I need to sleep and wake up earlier. It is a cycle. Again! The cycle is coming back in a few days. Finally, I do not exercise in the whole week.

(Student 17, clinical year, female)

\section{Fear that PA affect academic performance}

Some students believe that PA could be a cause of failure of academic performance.

I am focusing on my study. One senior student told me 'students, who exercise regularly, will fail the exam'. I do not want to be at risk. It is risky to exercise, just focus on the study.

(Student 3, preclinical year, female)

\section{DISCUSSION}

Less than half of the participants $(49.5 \%)$ were physically active. Male and preclinical students were more likely to be active. Recreational activity was a major type of PA among the medical students. Social support from friends and families could facilitate students to PA. Study-related activities and overtime shift work were the significant barriers to PA. The statistics showed preclinical students were more active than the clinical students. Men were likely to be active compared to women. According to the qualitative method, the clinical students had less free time. Their activities such as hospital attachment, ward round and night shift consumed a lot of time.

Compared with the Thai population $(71.6 \%$ were physically active), the participants were less active $(49.5 \%) .{ }^{20}$ In terms of energy use, the participants spent 540 MET-min/week (range 0-5640). The mean energy use of the Thai population was $7893 \pm 6527$ MET-min/ week. ${ }^{21}$ More than half of the participants were physically inactive. A similar trend was found in Turkish medical students. ${ }^{22}$ One study found the comparable results among medical college students to ours, and depicted that being a medical student was a risk of physical inactivity. ${ }^{23}$ A recent study showed that the level of PA among physicians, who regularly worked in the clinical practice, was low (only $31.6 \%$ are physically active) ${ }^{24}$ Alternatively, previous studies showed medical students had more participation in PA than the general populations, and more than two-thirds of medical students met the recommended levels of PA. ${ }^{15}$ The various PA levels among studies might reflect individual characteristics of each medical school. For instance, a lack of PA-friendly facilities and unsupportive policies may be reasons of insufficient PA among medical students.

According to the positive factors of PA, preclinical study and male gender were similar to findings in a study in Turkey. ${ }^{22}$ This study found that first year medical students were more active than final year students, and male medical students were more active than female students. ${ }^{22}$ Obviously, the preclinical students and clinical students had different lifestyles. The preclinical students often studied during working hours, from 08:00 to 16:00, and they had free time in the evening. It was a supportive ingredient to practice leisure PA. On the other hand, the clinical students could not predict the length of working hours, depending on their patients, seniors and workload. The female students were less active perhaps because they might have more personal barriers compared with the male students. For example, exposure to the sun light was a significant barrier to outdoor activities among Thai females due to cultural belief of the importance of fair skin. $^{25}$

In general, the associated factors of PA include environmental, social, psychosocial and cultural characteristics. ${ }^{26}$ The data from the in-depth interviews showed a variety of those characteristics among the medical students. The common findings, identified through the interviews, showed that medical students needed social support to participate in PA behaviours. Time limitations were considered a common barrier to PA. ${ }^{23}$ The in-depth data regarding time limitations was explored specifically and found that study-related activities and overtime shift work were significant issues. It is not unexpected that the clinical students were less active than the preclinical students because of the hospital attachments. Our findings explained that the medical students spent a lot of time in the hospitals, and the study-related activities consumed their free time. It was not only a loss of free time, but it was tiring.

The present study also identified the priority of academics, which was considered more important than participating in regular PA, as one of the barriers to PA. A study mentioned that reduction in exercise could reflect the stressful nature of medical training. ${ }^{28}$ It would be late to promote PA after the medical students had graduated because evidence has shown that clinicians were more active before starting graduate training. ${ }^{28}$ 
There were several strengths in this study. First, the response rate was high $(97.9 \%)$. Second, the study implemented a mixed method approach including the qualitative method to explore PA behaviours and factors influencing PA, which could explain the particular patterns among the medical students. Third, to ensure participants' understanding of PA definition, we provided the information regarding PA definition and examples on the questionnaires. Limitations included the small number of participants. Next, using a self-report questionnaire to collect PA participation is subjective and based on participants' recall memory. Therefore, we used the GPAQ, which is reliable and valid to reduce the risk of inaccurate data. ${ }^{29}{ }^{30}$ Finally, the findings were from the qualitative method based on a particular population which may not be generalised to other medical schools.

We recommend that there is a need to promote PA among medical students, particularly among clinical and female students. Both individual and population approaches are needed. For an individual approach, developing social support from friends and families may be a key tactic to increase PA. For a population approach, the medical school policymakers can take suitable action to highlight the importance of fellow students' and families' involvement in promoting PA. Finally, study-related barriers to PA can be balanced by supportive policies such as limiting the number of overtime shift per month.

\section{CONCLUSIONS}

Medical students in three Southern provinces of Thailand have a lower prevalence of the recommended levels of PA than the Thai population. Insufficient PA levels were identified in more than half of the medical students due to related barriers such as study-related activities and overtime shift work. As the less active populations, the clinical and female students should be a focused target to raise PA levels. An approach involving the medical school's policies should be an emphasis as a health promotion activity to balance study and health-related behaviours. Another approach among the medical students is carrying out the family's roles in promoting PA and developing peer support systems to initiate and maintain PA behaviours.

Acknowledgements The authors thank Dr Prachyapan Pethchuy, the Dean of School of Medicine, Walailak University for his permission to do this study; the Medical Education Centre of Trang Hospital and Vachira Phuket Hospital for their support; Professor Ng Chrik Jenn, University of Malaya, for his help as a manuscript proof reader; and Dr Lesley Dornan for English language editing.

Contributors AW contributed to the study design, conducted the research and prepared early drafts of the manuscript. KF, US and SV helped with the design of the study. US helped with the statistical analyses. KF helped with the qualitative analyses. All the authors read and approved the final version of the manuscript.

Funding This work was supported by the Institute of Research and Development, Walailak University (grant number WU59304).

Competing interests None declared.
Patient consent Obtained.

Ethics approval Ethical approval was acquired from the Human Research Ethics Committee Walailak University (protocol number 15/042).

Provenance and peer review Not commissioned; externally peer reviewed.

Data sharing statement No additional data are available.

Open Access This is an Open Access article distributed in accordance with the Creative Commons Attribution Non Commercial (CC BY-NC 4.0) license, which permits others to distribute, remix, adapt, build upon this work noncommercially, and license their derivative works on different terms, provided the original work is properly cited and the use is non-commercial. See: http:// creativecommons.org/licenses/by-nc/4.0/

\section{REFERENCES}

1. World Health Organization. Global status report on communicable diseases 2014. Geneva, Switzerland: World Health Organization, 2014.

2. Samitz G, Egger M, Zwahlen M. Domains of physical activity and all-cause mortality: systematic review and dose-response meta-analysis of cohort studies. Int J Epidemiol 2011;40:1382-400.

3. Caspersen CJ, Powell KE, Christenson GM. Physical activity, exercise, and physical fitness: definitions and distinctions for health-related research. Public Health Rep 1985;100:126-31.

4. World Health Organization. Global recommendations on physical activity for health. Geneva, Switzerland: World Health Organization, 2010.

5. Dumith SC, Hallal PC, Reis RS, et al. Worldwide prevalence of physical inactivity and its association with human development index in 76 countries. Prev Med 2011;53:24-8.

6. Al-Isa AN, Campbell J, Desapriya E, et al. Social and health factors associated with physical activity among Kuwaiti college students. J Obes 2011;2011:512363.

7. Bauman A, Bull F, Chey T, et al. The international prevalence study on physical activity: results from 20 countries. Int $J$ Behav Nutr Phys Act 2009;6:21.

8. Haase A, Steptoe A, Sallis JF, et al. Leisure-time physical activity in university students from 23 countries: associations with health beliefs, risk awareness, and national economic development. Prev Med 2004;39:182-90.

9. Irwin JD. Prevalence of university students' sufficient physical activity: a systematic review. Percept Mot Skills 2004;98(Pt 1):927-43.

10. Khalaf A, Ekblom Ö, Kowalski J, et al. Female university students' physical activity levels and associated factors-a cross-sectional study in southwestern Saudi Arabia. Int J Environ Res Public Health 2013;10:3502-17.

11. Korn L, Gonen E, Shaked $\mathrm{Y}$, et al. Health perceptions, self and body image, physical activity and nutrition among undergraduate students in Israel. PLOS ONE 2013;8:e58543.

12. Rao CR, Darshan B, Das N, et al. Practice of physical activity among future doctors: a cross sectional analysis. Int J Prev Med 2012;3:365-9.

13. Lobelo F, Duperly J, Frank E. Physical activity habits of doctors and medical students influence their counselling practices. Br J Sports Med 2009;43:89-92.

14. Stanford FC, Durkin MW, Stallworth JR, et al. Factors that influence physicians' and medical students' confidence in counseling patients about physical activity. J Prim Prev 2014;35:193-201.

15. Stanford FC, Durkin MW, Stallworth JR, et al. Comparison of physical activity levels in physicians and medical students with the general adult population of the United States. Phys Sportsmed 2013;41:86-92.

16. Wattanapisit A, Gaensan T, Anothaisintawee T, eds. Prevalence of physical activity and associated factors of medium and high activity among medical students at Ramathibodi Hospital. The 6th International Conference on Sport and Exercise Science 2015. Chonburi, Thailand: Institute of Physical Education, 2015.

17. World Health Organization. Global Physical Activity Questionnaire (GPAQ) Analysis Guide: World Health Organization. http://www.who. int/chp/steps/resources/GPAQ_Analysis_Guide.pdf (accessed 15 Jun 2015).

18. McLeroy KR, Bibeau D, Steckler A, et al. An ecological perspective on health promotion programs. Health Educ Q 1988;15:351-77.

19. Liamputtong P. Qualitative research methods. 4th edn. Melbourne: Oxford University Press, 2013. 
20. Division of Physical Activity and Health, Department of Health, Ministry of Public Health. Situation of physical activity/exercise among Thai people [in Thai]: Ministry of Public Health. https://sites. google.com/site/exercisemoph/sthankarn-kar-xxk-kalang-kay (accessed 15 Feb 2016).

21. Churangsarit S, Chongsuvivatwong V. Spatial and social factors associated with transportation and recreational physical activity among adults in Hat Yai City, Songkhla, Thailand. J Phys Act Health 2011;8:758-65.

22. Nacar M, Cetinkaya F, Baykan Z, et al. Hazardous health behaviour among medical students: a study from Turkey. Asian Pac J Cancer Prev 2015;16:7675-81.

23. Awadalla NJ, Aboelyazed AE, Hassanein MA, et al. Assessment of physical inactivity and perceived barriers to physical activity among health college students, south-western Saudi Arabia. East Mediterr Health J 2014;20:596-604.

24. Al Reshidi FS. Level of physical activity of physicians among residency training program at Prince Sultan Military Medical City, Riyadh, KSA 2014. Int J Health Sci (Qassim) 2016;10(1):39-47.

25. Sims $\mathrm{CH}$. Genderized workplace lookism in the U.S. and abroad: implications for organization and career development professionals.
In: Hughes C, ed. Impact of diversity on organization and career development. Pennsylvania Business Science Reference, 2015:105-28.

26. Saimon R, Choo WY, Bulgiba A. "Feeling unsafe": a photovoice analysis of factors influencing physical activity behaviour among Malaysian adolescents. Asia Pac J Public Health 2015;27: NP2079-92.

27. Saimon R, Choo WY, Chang KH, et al. Physical activity among adolescents in an East Malaysian rural indigenous community: exploring the influence of neighborhood environmental factors. Asia Pac J Public Health 2015;27(8 Suppl):33S-40S.

28. Gnanendran A, Pyne DB, Fallon KE, et al. Attitudes of medical students, clinicians and sports scientists towards exercise counselling. J Sports Sci Med 2011;10:426-31.

29. Bull FC, Maslin TS, Armstrong T. Global Physical Activity Questionnaire (GPAQ): nine country reliability and validity study. $J$ Phys Act Health 2009;6:790-804.

30. Cleland CL, Hunter RF, Kee F, et al. Validity of the Global Physical Activity Questionnaire (GPAQ) in assessing levels and change in moderate-vigorous physical activity and sedentary behaviour. BMC Public Health 2014;14:1255. 